\title{
COMPLETE SLICES AND HOMOLOGICAL PROPERTIES OF TILTED ALGEBRAS
}

\author{
by IBRAHIM ASSEM and FLÁVIO ULHOA COELHO
}

(Received 23 February, 1993)

1. Introduction. It is reasonable to expect that the representation theory of an algebra (finite dimensional over a field, basic and connected) can be used to study its homological properties. In particular, much is known about the structure of the Auslander-Reiten quiver of an algebra, which records most of the information we have on its module category. We ask whether one can predict the homological dimensions of a module from its position in the Auslander-Reiten quiver. We are particularly interested in the case where the algebra is a tilted algebra. This class of algebras of global dimension two, introduced by Happel and Ringel in [7], has since then been the subject of many investigations, and its representation theory is well understood by now (see, for instance, [1], [7], [8], [9], [11], [13]). In this case, the most striking feature of the Auslander-Reiten quiver is the existence of complete slices, which reproduce the quiver of the hereditary algebra from which the tilted algebra arises. It follows from well-known results that any indecomposable successor (or predecessor) of a complete slice has injective (or projective, respectively) dimension at most one, from which one deduces that a tilted algebra is representation-finite if and only if both the projective and the injective dimensions of almost all (that is, all but at most finitely many non-isomorphic) indecomposable modules equal two (see (3.1) and (3.2)). On the other hand, the authors have shown in [2, (3.4)] that a representation-infinite algebra is concealed if and only if both the projective and the injective dimensions of almost all indecomposable modules equal one (see also [14]). This leads us to consider, for tilted algebras which are not concealed, the case when the projective (or injective) dimension of almost all indecomposable successors (or predecessors, respectively) of a complete slice equal two. In order to answer this question, we define the notions of left and right type of a tilted algebra, then those of reduced left and right types (see (2.2) and (3.4) for the definitions). Our main theorem is as follows.

THEOREM. Let $A$ be a representation-infinite algebra which is tilted but not concealed, and $\Sigma$ a complete slice in its Auslander-Reiten quiver $\Gamma(\bmod A)$.

(a) $\operatorname{pd} M=2$ for almost all indecomposable successors $M$ of $\Sigma$ if and only if the reduced right type of $A$ is empty or a disjoint union of Dynkin graphs.

(b) id $M=2$ for almost all indecomposable predecessors $M$ of $\Sigma$ if and only if the reduced left type of $A$ is empty or a disjoint union of Dynkin graphs.

Our paper is organised as follows. In Section 2, after setting the notation and briefly recalling some definitions and results that will be needed in the sequel, we introduce the notions of left and right type and show some of their properties, in particular those related to the left and right end algebras introduced by Kerner in [8]. In Section 3, we discuss some of the above-mentioned homological properties, define the notions of reduced left and right types, and then prove our main theorem.

Glasgow Math. J. 36 (1994) 347-354. 


\section{The left and right types of a tilted algebra.}

2.1. Notation. All algebras in this paper are basic, connected, associative, finite dimensional algebras with identities over a fixed algebraically closed field $k$, and all modules are finitely generated right modules. For an algebra $A$, we denote by $\bmod A$ its module category, and by ind $A$ a full subcategory of $\bmod A$ consisting of a complete set of representatives of the isomorphism classes of indecomposable objects in $\bmod A$. We shall use freely and without further reference properties of $\bmod A$, the Auslander-Reiten translations $\tau=\mathrm{DTr}$ and $\tau^{-1}=\operatorname{TrD}$, and the Auslander-Reiten quiver $\Gamma(\bmod A)$ of $A$ as can be found, for instance, in [3], [11]. A path $x_{0} \rightarrow x_{1} \rightarrow \ldots \rightarrow x_{m}$ in $\Gamma(\bmod A)$ is called sectional if $\tau x_{i+1} \neq x_{i-1}$ when $0<i<m$, and a full subquiver $\Sigma$ of $\Gamma(\bmod A)$ is called a subsection if it is connected and each path in $\Sigma$ is sectional [5]. Given an $A$-module $M$, we denote by $\operatorname{pd} M$ its projective dimension and by id $M$ its injective dimension.

For tilting theory, we refer the reader to [1], [11]. In particular, we recall that tilted algebras are characterised by the existence of complete slices in a component of their Auslander-Reiten quiver, called connecting component [11, (4.2)]. A tilted algebra has at most two connecting components and, if it has two, then it is a concealed algebra [12, Lecture 2]. We recall the notion of type of a tilted algebra (see, for instance, $[1,(5.1)]$ ). Let $A$ be a tilted algebra. There exists a finite connected quiver $\Sigma$ without oriented cycles and a tilting module $T$ over the path algebra $k \Sigma$ of $\Sigma$ such that $A=$ End $T$. Clearly, this is equivalent to the requirement that $\Gamma(\bmod A)$ contains a complete slice whose underlying quiver is isomorphic to $\Sigma^{\text {op }}$. The quiver $\Sigma$ is generally not uniquely determined by $A$, but two different quivers $\Sigma$ and $\Sigma^{\prime}$ whose path algebras tilt to $A$ have the same underlying graph and can be deduced from each other by an admissible change of orientation (that is, a sequence of reflections). The underlying graph $\bar{\Sigma}$ of a quiver $\Sigma$ whose path algebra tilts to $A$ is called the type of $A$ and a complete slice in $\Gamma(\bmod A)$ whose underlying quiver is isomorphic to $\Sigma^{\mathrm{op}}$ is called a representing slice.

2.2. Let now $A$ be a tilted algebra. We shall define the notions of left and right type of $A$, using the description of $\bmod A$ given in $[8,(4.1)]$.

We define the left type of $A$ as follows. If $A$ has a complete slice in a postprojective component (following [6], we use the term postprojective rather than preprojective: we believe it is more descriptive), the left type of $A$ is defined to be the empty graph. Otherwise, $A$ has a unique connecting component $\Gamma$ which is not postprojective. If $\Gamma$ contains no projective module (so that every module in $\Gamma$ is left stable), we define the left type of $A$ to be the type of the tilted algebra $A$, as defined above. Suppose $\Gamma$ contains a projective module. Let $\Sigma$ be the subsection of $\Gamma$ consisting of the left stable modules $M$ in $\Gamma$ such that there exists a path in $\Gamma$ of length at least one from $M$ to some projective, and any such path is sectional. Since $\Sigma$ is generally not connected, we can write it as $\Sigma_{1} \cup \ldots \cup \Sigma_{t}$, where each $\Sigma_{i}$ is a connected component of $\Sigma$. Then $\Sigma=\Sigma_{1} \cup \ldots \cup \Sigma_{t}$ will be called the left extremal subsection of $A$, and its underlying graph $\bar{\Sigma}$ will be called the left type of $A$. Observe that, since $\Gamma$ contains a complete slice, no injective module is a predecessor of $\Sigma$.

Dually, we define the right type of $A$ as follows. If $A$ has a complete slice in a preinjective component, the right type of $A$ is defined to be the empty graph. Otherwise, $A$ has a unique connecting component $\Gamma$ which is not preinjective. If $\Gamma$ contains no injective module (so that every module in $\Gamma$ is right stable), we define the right type of $A$ to be the type of the tilted algebra $A$. If $\Gamma$ contains an injective module, let $\Sigma$ be the 
subsection of $\Gamma$ consisting of the right stable modules $M$ in $\Gamma$ such that there exists a path in $\Gamma$ of length at least one from some injective to $M$, and any such path is sectional. Let $\Sigma=\Sigma_{1} \cup \ldots \cup \Sigma_{t}$, where each $\Sigma_{i}$ is a connected component of $\Sigma$. Then $\Sigma=\Sigma_{1} \cup \ldots \cup \Sigma_{t}$ will be called the right extremal subsection of $A$, and its underlying graph $\bar{\Sigma}$ will be called the right type of $A$. Observe that, since $\Gamma$ contains a complete slice, no projective module is a successor of $\Sigma$.

These notions may also be interpreted in terms of the left and right stable parts of an Auslander-Reiten component (see [10]). Let $A$ be an arbitrary algebra and let $\Gamma$ be a component of $\Gamma(\bmod A)$. We define the left stable part of $\Gamma$ to be the full subquiver $\Gamma_{l}$ consisting of the indecomposable modules $M$ in $\Gamma$ such that:

(i) $M$ is left stable, that is, $\tau^{t} M \neq 0$ for all $t \geq 0$, and

(ii) $M$ is not a successor of a projective module in $\Gamma$.

Dually, the right stable part of $\Gamma$ is defined to be the full subquiver $\Gamma_{r}$ consisting of the indecomposable modules $M$ in $\Gamma$ such that:

(i) $M$ is right stable, that is, $\tau^{-s} M \neq 0$ for all $s \geq 0$, and

(ii) $M$ is not a predecessor of an injective module in $\Gamma$.

If now $A$ is a tilted algebra, and $\Gamma$ is a connecting component in $\Gamma(\bmod A)$ it follows from $[8,(4.1)]$ that $\Gamma_{1}$ is a disjoint union of connected translation quivers, each of which consists of all predecessors of a complete slice lying in the preinjective component of a tilted algebra. Thus, if $\Gamma$ is a postprojective component, then $\Gamma_{l}$ is empty (and so is the left type) while, if $\Gamma$ is not a postprojective component, the left type of $A$ is the underlying graph of a disjoint union of complete slices in $\Gamma_{l}$. Dually, if $\Gamma$ is a preinjective component, then $\Gamma_{r}$ is empty (and so is the right type) while, if $\Gamma$ is not a preinjective component, the right type of $A$ is the underlying graph of a disjoint union of complete slices in $\Gamma_{r}$.

Example. We borrow this example from $[8,(5)]$. Let $A$ be given by the quiver

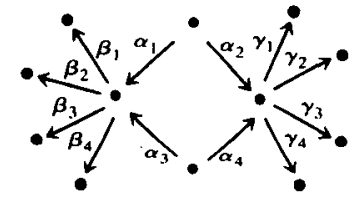

bound by $\alpha_{1} \beta_{i}=0=\alpha_{2} \gamma_{i}$ for $i=1,2,3$ and $\alpha_{3} \beta_{j}=0=\alpha_{4} \gamma_{j}$ for $j=2,3,4$. Then $A$ is tilted, and its type is the underlying graph of the following representing slice

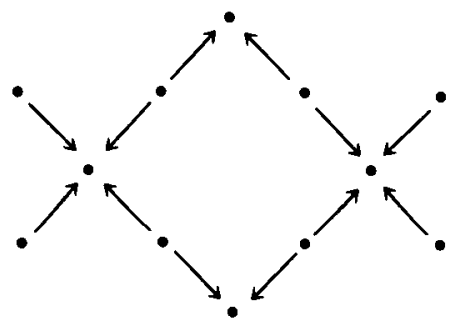

The left type of $A$ equals the disjoint union of two copies of $\tilde{D}_{4}$, and the left extremal subsection equals the disjoint union of two copies of the quiver

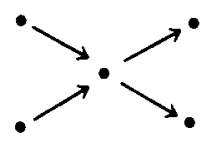


The right type of $A$ is equal to $\tilde{A}_{7}$, and the right extremal subsection is given by the quiver

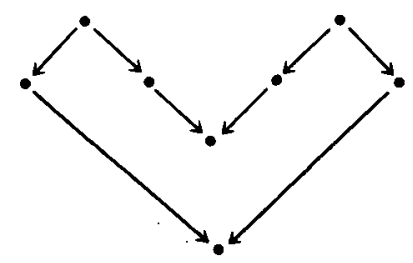

2.3. The following lemma records the most immediate properties of the left and right types of a tilted algebra.

Lemma. Let $A$ be a tilted algebra.

(a) The left and the right types of $A$ are empty graphs if and only if $A$ is representation-finite or concealed.

(b) The left (or right) type of $A$ equals the type of $A$ if and only if $A$ has a unique connecting component containing no projective (or no injective, respectively).

(c) The left type and the right type of $A$ are both equal to the type of $A$ if and only if $A$ is the endomorphism algebra of a regular tilting module over a wild hereditary algebra.

(d) If $A$ is not concealed, $A$ is tame if and only if the left and the right types of $A$ are empty or disjoint unions of euclidean graphs.

Proof. (a) The left type of $A$ is empty if and only if $A$ has a complete slice in a postprojective component $\Gamma$, and the right type of $A$ is empty if and only if $A$ has a complete slice in a preinjective component $\Gamma^{\prime}$. If $\Gamma=\Gamma^{\prime}$, then $A$ is representation-finite. If $\Gamma \neq \Gamma^{\prime}$, then $A$ is concealed. The converse is obvious.

(b) Follows directly from the definition.

(c) Applying (b), the left type of $A$, its right type and its type are equal if and only if $A$ has a unique connecting component containing neither projectives nor injectives, that is, which is stable or, equivalently, which is of the form $\mathbb{Z} \Sigma$ for some quiver $\Sigma$. This is the case if and only if $A$ is the endomorphism algebra of a tilting $k \Sigma$-module with neither postprojective nor preinjective direct summands, that is, which is regular (see $[\mathbf{1 2},(1)]$ ).

(d) This follows from the description of $\Gamma(\bmod A)$ given in [8]. Indeed, if the right type and the left type of $A$ are disjoint unions of euclidean graphs, then any component of $\Gamma(\bmod A)$ which precedes or succeeds a complete slice is a component of a tilted algebra of euclidean type. Hence $A$ is tame and even domestic. The converse is equally easy.

2.4. Of more immediate concern to us, however, is the relation between the left and right types and the left and right end algebras as defined in [8]. We record it in the following lemma.

Lemma. Let $A$ be a representation-infinite algebra which is tilted but not concealed.

(a) Each connected component of the left extremal subsection is a complete slice in the connecting component without projective modules of the Auslander-Reiten quiver of a connected component of the left end algebra ${ }_{\infty} A$. In particular, the left type of $A$ equals the type of ${ }_{\infty} A$ as a tilted algebra.

(b) Each connected component of the right extremal subsection is a complete slice in 
the connecting component without injective modules of the Auslander-Reiten quiver of a connected component of the right end algebra $A_{x}$. In particular, the right type of $A$ equals the type of $A_{x}$ as a tilted algebra.

Proof. Under the stated hypothesis, $A$ has a unique connecting component. The statement then follows from the description of $\bmod A$ as given in [8], and the respective definitions.

\section{The main result.}

3.1. We start our discussion with the following lemma, which is a direct consequence of well-known homological properties of tilting modules (see, for instance, [1, (2.2)]). For the convenience of the reader, we shall however provide a (simple) direct proof.

Lemma. Let $A$ be a tilted algebra and $\Sigma$ a complete slice in $\Gamma(\bmod A)$.

(a) If $M$ is an indecomposable successor of $\Sigma$, then id $M \leq 1$.

(b) If $M$ is an indecomposable predecessor of $\Sigma$, then $\mathrm{pd} M \leq 1$.

Proof. We shall only prove (a), since the proof of (b) is dual. Let $T=\bigoplus\{N: N \in \Sigma\}$. Assume id $M>1$ for some indecomposable successor $M$ of $\Sigma$. Then $\operatorname{Hom}_{A}\left(\tau^{-1} M, A\right) \neq 0$ (by $\left[11,(2.4)\left(1^{*}\right)\right.$ p. 74]). Let $P_{A}$ be an indecomposable projective module such that $\operatorname{Hom}_{A}\left(\tau^{-1} M, P\right) \neq 0$. Since $M$ is a successor of $\Sigma$, so are $\tau^{-1} M$ and $P$. Hence $P$ belongs to the subcategory of all $A$-modules generated by $T$ so that there exist $m>0$ and an epimorphism $T^{(m)} \rightarrow P$ which must split, because $P$ is projective. Hence $P$ is a direct summand of $T$, and so belongs to $\Sigma$. We have obtained a sequence of non-zero non-isomorphisms $U \rightarrow \ldots \rightarrow M \rightarrow * \rightarrow \tau^{-1} M \rightarrow P$ with both $U, P$ on $\Sigma$, which is not sectional, a contradiction to the fact that $\Sigma$ is a complete slice.

3.2. As a consequence of this lemma and the fact that the global dimension of a tilted algebra is at most two, we have the following result.

Corollary. $A$ tilted algebra $A$ is representation-finite if and only if $\operatorname{pd} M=2$ and id $M=2$ for almost all $M$ in ind $A$.

Proof. Since the necessity is obvious, let us show the sufficiency. Since $A$ is tilted, there exists a complete slice $\Sigma$ in $\Gamma(\bmod A)$. By $(3.1), \Sigma$ has only finitely many predecessors and only finitely many successors. Hence $A$ is representation-finite.

3.3. The following result was obtained in.[2, (3.4)] as a consequence of the theory of postprojective and preinjective partitions of [4], and independently in [14].

Proposition. $A$ representation-infinite algebra $A$ is concealed if and only if $\mathrm{pd} M=1$ and id $M=1$ for almost all $M$ in ind $A$.

3.4. We shall now define the reduced right and left types of a representation-infinite tilted algebra $A$.

Let $A$ be a representation-infinite tilted algebra. We first define the reduced left type of $A$. If $A$ has a postprojective component containing a complete slice or if the unique connecting component of $A$ contains no projective module, we define the reduced left type of $A$ to be equal to the left type of $A$, that is, respectively, the empty graph and the type of $A$. Assume that the unique connecting component of $A$ is not postprojective but contains projectives, and let $\Sigma=\Sigma_{1} \cup \ldots \cup \Sigma_{r}$ be the left extremal subsection of $A$. We 
define the reduced left extremal subsection of $A$ to be $\Sigma_{1}^{\prime} \cup \ldots \cup \Sigma_{t}^{\prime}$ where, for each $i, \Sigma_{i}^{\prime}$ is the full (convex) subquiver of $\Sigma_{i}$ obtained by deleting all the sinks. The reduced left type of $A$ is then the underlying graph $\bar{\Sigma}_{1}^{\prime} \cup \ldots \cup \bar{\Sigma}_{t}^{\prime}$ of the reduced left extremal subsection. Observe that the sinks of $\Sigma_{i}$ correspond to radical summands of projective $A$-modules.

Likewise, we define the reduced right type of $A$. If $A$ has a preinjective component containing a complete slice or if the unique connecting component of $A$ contains no injective module, we define the reduced right type of $A$ to be equal to the right type of $A$, that is, respectively, the empty graph and the type of $A$. Assume that the unique connecting component of $A$ is not preinjective but contains injectives, and let $\Sigma=\Sigma_{1} \cup \ldots \cup \Sigma_{t}$ be the right extremal subsection of $A$. We define the reduced right extremal subsection of $A$ to be $\Sigma_{1}^{\prime} \cup \ldots \cup \Sigma_{i}^{\prime}$ where, for each $i, \Sigma_{i}^{\prime}$ is the full (convex) subquiver of $\Sigma_{i}$ obtained by deleting all the sources. The reduced right type of $A$ is then the underlying graph $\bar{\Sigma}_{1}^{\prime} \cup \ldots \cup \bar{\Sigma}_{t}^{\prime}$ of the reduced right extremal subsection. Observe that the sources of $\Sigma_{i}$ correspond to socle factors of injective $A$-modules.

Since $A$ is representation-infinite, the reduced left (or right) type of $A$ is empty if and only if so is the left (or right, respectively) type of $A$.

For instance, in the example in (2.2), the reduced left extremal subsection is the disjoint union of two copies of the quiver

while the reduced right extremal subsection is the disjoint union of two copies of the quiver

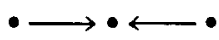

In particular, both the reduced right and left types of $A$ are disjoint unions of Dynkin graphs, so that $A$ satisfies the conditions of our main theorem below.

THEOREM. Let $A$ be a representation-infinite algebra which is tilted but not concealed, and $\Sigma$ a complete slice in $\Gamma(\bmod A)$.

(a) $\operatorname{pd} M=2$ for almost all indecomposable successors $M$ of $\Sigma$ if and only if the reduced right type of $A$ is empty or a disjoint union of Dynkin graphs.

(b) id $M=2$ for almost all indecomposable predecessors $M$ of $\Sigma$ if and only if the reduced left type of $A$ is empty or a disjoint union of Dynkin graphs.

Proof. We shall only prove (a), since the proof of (b) is dual.

(i) Proof of sufficiency. Since $A$ is not concealed, there exists only one component $\Gamma$ containing complete slices. Now, if the reduced right type is empty then $\Gamma$ is preinjective, $\Sigma$ has at most finitely many non-isomorphic indecomposable successors, and clearly, the projective dimension of almost all of them equals 2. Suppose now that the reduced right type of $A$ is a non-empty disjoint union of Dynkin graphs, which is equivalent, in our case, to saying that $\Gamma$ is not a preinjective component. Since the global dimension of $A$ is at most 2 and pd $M>1$ if and only if $\operatorname{Hom}_{A}(\mathrm{D} A, \tau M) \neq 0$ (by [11, (2.4)(1) p. 74]), it suffices to prove that $\operatorname{Hom}_{A}(\mathrm{D} A, N) \neq 0$ for almost all indecomposable successors $N$ of $\Sigma$. Let thus $A_{\infty}=A_{1} \times \ldots \times A_{i}$, where the $A_{i}$ are connected tilted algebras. For each $i$, let $\Sigma_{i}$ be the right extremal subsection in the connecting component of $\Gamma\left(\bmod A_{i}\right)$ as 
constructed in (2.2), so that the right type of $A$ equals $\bar{\Sigma}_{1} \cup \ldots \cup \bar{\Sigma}_{t}$ (by (2.4)). Let $T=\bigoplus\left\{M: M \in \Sigma_{i}\right\}$, considered as an $A_{i}$-module. Then $H=\operatorname{End}\left(T_{A_{i}}\right)$ is a hereditary algebra and, for each source $S$ in $\Sigma_{i}$, the $H$-module $S^{\prime}=\operatorname{Hom}_{A_{i}}(T, S)$ is simple projective.

Let $U$ denote the direct sum of all sources in $\Sigma_{i}$ and set $U^{\prime}=\operatorname{Hom}_{A_{i}}(T, U)$. Since $U^{\prime}$ is the direct sum of simple projective $H$-modules, we can write $U^{\prime}=e H$ for some non-zero idempotent $e \in H$. The hereditary algebra $H^{\prime}=\operatorname{End}(1-e) H$ has for type the full convex subquiver $\Sigma_{i}^{\prime}$ of $\Sigma_{i}$ obtained by dropping the summands of $U$. That is, $\Sigma_{i}^{\prime}$ is the (disjoint union of the) component(s) of the reduced type corresponding to $\Sigma_{i}$, hence $\bar{\Sigma}_{i}^{\prime}$ is a (disjoint union of) Dynkin graph(s), by hypothesis. Consequently, $H^{\prime}$ is representationfinite. This implies that $\operatorname{Hom}_{H}\left(U^{\prime}, X\right) \neq 0$ for almost all indecomposable $H$-modules $X$.

Let $(\mathscr{T}, \mathscr{F})$ denote the torsion theory induced by $T$ in $\bmod A_{i}$, that is, let $\mathscr{T}$ be the class of all $A_{i}$-modules generated by $T$, while $\mathscr{F}$ consists of the modules $X$ such that $\operatorname{Hom}_{A_{i}}(T, X)=0$. It is easy to see that $\mathscr{F}$ consists of the proper predecessors of $\Sigma_{i}$ in the connecting component of $\Gamma\left(\bmod A_{i}\right)$, hence contains only finitely many non-isomorphic indecomposables. On the other hand, by the Brenner-Butler theorem,

$$
\operatorname{Hom}_{A_{i}}(U, M) \cong \operatorname{Hom}_{H}\left(U^{\prime}, \operatorname{Hom}_{A_{i}}(T, M)\right)
$$

for any $M$ in $\mathscr{T}$. This implies that $\operatorname{Hom}_{A_{i}}(U, M) \neq 0$ for almost all $M$ in ind $A_{i}$.

Since, for any indecomposable summand $S$ of $U$, there exists an indecomposable injective $A$-module $I$ and in irreducible epimorphism $I \rightarrow S$, we deduce that $\operatorname{Hom}_{A}(\mathrm{D} A, N) \neq 0$ for almost all $N$ in ind $A_{i}$. This being true for each $i$, we infer that $\operatorname{Hom}_{A}(\mathrm{D} A, N) \neq 0$ for almost all $N$ in ind $A_{\infty}$. Since almost all indecomposable successors of $\Sigma$ are $A_{\infty}$-modules, this completes the proof of the sufficiency.

(ii) Proof of necessity. Suppose that $A$ is a representation-infinite tilted algebra and let $\Sigma$ be a complete slice in a connecting component $\Gamma$ of $\Gamma(\bmod A)$ such that $\operatorname{pd} M=2$ for almost all indecomposable successors $M$ of $\Sigma$. If $\Gamma$ is a preinjective component then the reduced right type of $A$ is the empty graph and we are done. Suppose from now on that $\Gamma$ is not a preinjective component and let $\Sigma_{1} \cup \ldots \cup \Sigma_{\mathrm{t}}$ denote the right extremal subsection of $A$, where each $\Sigma_{i}$ is connected. We must show that the reduced right extremal subsection $\Sigma_{1}^{\prime} \cup \ldots \cup \Sigma_{t}^{\prime}$ is such that, when $1 \leq i \leq t, \bar{\Sigma}_{i}^{\prime}$ is a (disjoint union of) Dynkin graph(s). Assume that, for some $i, \bar{\Sigma}_{i}^{\prime}$ is not a (disjoint union of) Dynkin graph(s) and let $H^{\prime}$ be the endomorphism algebra of the module $\bigoplus\left\{M: M \in \Sigma_{i}^{\prime}\right\}$. Then $H^{\prime}$ is a representation-infinite full convex subcategory of the (hereditary) endomorphism algebra $H$ of the module $\bigoplus\left\{N: N \in \Sigma_{i}\right\}$. By definition (and (2.4)), the types of $A_{i}$ (which is the connected component of $A_{\infty}$ having $\Sigma_{i}$ as a complete slice in its connecting component) and of $H$ coincide and equal $\bar{\Sigma}_{i}$. Moreover, there are infinitely many non-isomorphic indecomposable $A_{i}$-modules $\left(L_{\lambda}\right)_{\lambda \in \Lambda}$ such that $\operatorname{Hom}_{A}\left(S_{1} \oplus \ldots \oplus S_{m}, L_{\lambda}\right)=0$ for all $\lambda \in \Lambda$, where $S_{1}, \ldots, S_{m}$ are all the sources in $\Sigma_{i}$. We claim that this implies $\operatorname{Hom}_{A}\left(\mathrm{D} A, L_{\lambda}\right)=0$ for all $\lambda \in \Lambda$. Indeed, let $I$ be an indecomposable injective $A$-module and consider the left minimal almost split morphism $f: I \rightarrow K$. If $K$ has no summand in $\bmod A_{i}$, then clearly $\operatorname{Hom}_{A}\left(I, L_{\lambda}\right)=0$ since any non-zero morphism would factor through $f$, an absurdity. If $K$ has a summand in $\bmod A_{i}$, it must be one of $S_{1}, \ldots, S_{m}$. Thus, $\operatorname{Hom}_{A}\left(I, L_{\lambda}\right) \neq 0$ implies $\operatorname{Hom}_{A}\left(S_{j}, L_{\lambda}\right) \neq 0$ for some $j$ such that $1 \leq j \leq m$, a contradiction to our assumption on the family $\left(L_{\lambda}\right)_{\lambda \in \Lambda}$. This shows that we indeed have $\operatorname{Hom}_{A}\left(\mathrm{D} A, L_{\lambda}\right)=0$ for all $\lambda \in \Lambda$, or, equivalently, that $\operatorname{pd}\left(\tau^{-1} L_{\lambda}\right) \leq 1$ for all $\lambda \in \Lambda$, a contradiction to our hypothesis. 
COROLlaRY. Let $A$ be a representation-infinite tilted algebra.

(a) If $A$ has a complete slice in a postprojective component then $p d M=2$ for almost all $M$ in ind $A$ if and only if the reduced right type of $A$ is a disjoint union of Dynkin graphs.

(b) If $A$ has a complete slice in a preinjective component then id $M=2$ for almost all $M$ in ind $A$ if and only if the reduced left type of $A$ is a disjoint union of Dynkin graphs.

ACKNOWLEDGEMENTS. This paper was written while the second author was visiting the first. The authors gratefully acknowledge partial support from NSERC (Canada), FAPESP (Brasil) and the Université de Sherbrooke.

\section{REFERENCES}

1. I. Assem, Tilting theory - an introduction, in Topics in algebra, Banach Centre Publications 26 (PWN, 1990), 127-180.

2. I. Assem and F. U. Coelho, Glueings of tilted algebras, J. Pure Appl. Algebra, to appear.

3. M. Auslander and I. Reiten, Representation theory of Artin algebras. III, almost split sequences, and IV, invariants given by almost split sequences, Comm. Algebra 3 (1975), 239-294 and 5 (1977), 443-518.

4. M. Auslander and S. O. Smalø, Preprojective modules over Artin algebras, J. Algebra 66 (1980), 61-122.

5. R. Bautista, Sections in Auslander-Reiten quivers, Proc. ICRA II (Ottawa, 1979), Lecture Notes in Mathematics 832 (Springer, 1980), 74-96.

6. P. Gabriel and A. V. Rojter, Representations of finite-dimensional algebras, Encyclopaedia of Mathematical Sciences 73, Algebra VIII (Springer, 1992).

7. D. Happel and C. M. Ringel, Tilted algebras, Trans. Amer. Math. Soc. 274 (1982), 399-443.

8. O. Kerner, Tilting wild algebras, J. London Math. Soc. (2) 39 (1989), 29-47.

9. S. Liu, The connected components of the Auslander-Reiten quiver of a tilted algebra, $J$. Algebra 161 (1993), 505-523.

10. S. Liu, Semi-stable components of an Auslander-Reiten quiver, J. London Math. Soc. (2) 47 (1993), 405-416.

11. C. M. Ringel, Tame algebras and integral quadratic forms, Lecture Notes in Mathematics 1099 (Springer, 1984).

12. C. M. Ringel, Representation theory of finite-dimensional algebras, in Representations of algebras (Durham, 1985), London Math. Soc. Lecture Notes Series 116 (Cambridge University Press, 1986) 7-79.

13. C. M. Ringel, The regular components of the Auslander-Reiten quiver of a tilted algebra, Chinese Ann. Math. Ser. B 9 (1988), 1-18.

14. A. Skowroński, Minimal representation-infinite artin algebras, Math. Proc. Cambridge Philos. Soc., to appear.

MATHÉMATIQUES ET INFORMATIQUE

UNIVERSITÉ DE SHERBROOKE

SHERBROOKE

QUEBEC J1K 2R1

Canada

E-mail address: iassem@vml.si.usherb.ca
Departamento de Matemática-IME

Universidade de São PaUlo

CP 20570

São Paulo

SP, 01452-990

BrasiL

E-mail address: fucoelho@ime.usp.br 